\title{
Integrated scenario with type-III ELMy H-mode edge: extrapolation to ITER
}

\author{
J. Rapp ${ }^{1,2}$, Y. Corre ${ }^{3}$, Y. Andrew ${ }^{4}$, M.R. de Baar ${ }^{1}$, M. Beurskens ${ }^{4}$, \\ S. Brezinsek ${ }^{2}$, M. Brix ${ }^{2}$, S. Devaux ${ }^{5}$, T. Eich ${ }^{5}$, R. Felton ${ }^{4}$, W. Fundamenski ${ }^{4}$, \\ C. Giroud ${ }^{4}$, D. Howell ${ }^{4}$, A. Huber ${ }^{2}$, S. Jachmich ${ }^{6}$, E. Joffrin ${ }^{3}$, A. Korotkov ${ }^{4}$, \\ G.F. Matthews ${ }^{4}$, D.C. McDonald ${ }^{4}$, A. Meigs ${ }^{4}$, P. Monier-Garbet ${ }^{3}$, \\ P. Morgan $^{4}$, I. Nunes ${ }^{7}$, G.J. van Rooij ${ }^{1}$, O. Sauter ${ }^{8}$, M.F. Stamp ${ }^{4}$, \\ G. Telesca ${ }^{6}$, P.C. de Vries $^{4}$, R. Zagorski ${ }^{9}$ and JET-EFDA contributors ${ }^{a}$ \\ JET-EFDA, Culham Science Centre, OX14 3DB, Abingdon, UK \\ ${ }^{1}$ FOM Instituut voor Plasma Fysica Rijnhuizen, EURATOM Association, TEC, Nieuwegein, The \\ Netherlands \\ 2 IEF-4, Forschungszentrum Jülich GmbH, EURATOM Association, TEC, Jülich, Germany \\ ${ }^{3}$ Association EURATOM-CEA sur la Fusion Controlee, Cadarache, Saint-Paul-lez-Durance, France \\ ${ }^{4}$ EURATOM-UKAEA/Fusion Association, Culham Science Center, Abingdon, OXON, UK \\ ${ }^{5}$ Max-Planck Institut für Plasmaphysik, EURATOM Association, Garching, Germany \\ ${ }^{6}$ LPP-ERM/KMS, EURATOM-Belgian State Association, TEC, Brussels, Belgium \\ ${ }^{7}$ Association EURATOM/IST, Centro de Fusao Nuclear, Lisbon, Portugal \\ ${ }^{8}$ CRPP, Association EURATOM-Confederation Suisse, EPFL, Lausanne, Switzerland \\ ${ }^{9}$ Institute of Plasma Physics and Laser Microfusion, EURATOM Association, Warsaw, Poland
}

Received 29 December 2008, accepted for publication 7 July 2009

Published 14 August 2009

Online at stacks.iop.org/NF/49/095012

\begin{abstract}
One of the most severe problems for fusion reactors is the power load on the plasma facing components. The challenge is to develop operation scenarios, which combine sufficient energy confinement with benign heat loads to the plasma facing components. The radiative type-III ELMy H-mode seems a possible solution for such an integrated ITER scenario. Nitrogen seeded type-III ELMy H-modes for the standard inductive scenario and the high beta stationary hybrid scenario are investigated with respect to their transient and steady-state power fluxes to the divertor, confinement properties, edge operational space, core operational space, plasma purity and MHD behaviour. A large database of highly radiative type-III ELMy H-modes on JET is used for extrapolations to ITER. On this basis the transient heat load should be acceptable for ITER. It was found that the scaling of the confinement time with respect to the ion gyroradius is close to the gyro-Bohm scaling. Scalings with respect to the plasma collisionality suggest that the confinement will be good enough for an ITER scenario at 17 MA with a power amplification factor $(Q)$ of 10 and might be marginally good enough for a $Q=10$ scenario at $15 \mathrm{MA}$. Those extrapolations are supported by simulations with an integrated core/edge model COREDIV. In addition the hybrid scenario with type-III edge localized modes has been proven to have improved edge conditions without any modification of the central plasma current profile, indicating it is compatible with a high beta operation for a steady-state ITER $Q=5$ scenario.
\end{abstract}

PACS numbers: 52.25.Vy, 52.40.Hf, 52.55.Fa, 52.55.Rk

(Some figures in this article are in colour only in the electronic version)

\section{Introduction}

One of the most severe problems for fusion reactors is the power load on the plasma facing components. Technically only loads of less than $10 \mathrm{MW} \mathrm{m}^{-2}$ in steady state and less than $0.5 \mathrm{MJ} \mathrm{m}^{-2}$ [1] during transients, caused by the so-called

a See appendix of the paper by F. Romanelli 2008 Overview of JET results Proc. IAEA Conf. on Fusion Energy (Geneva, Switzerland, 2008). edge localized modes (ELMs) [2], which are an integral part of the H-mode [3], are acceptable. Predictions of the transient heat flux due to type-I ELMs $[4,5]$ lead to the conclusion that unmitigated type-I ELMs will be most likely not acceptable for ITER. Currently, alternative scenarios are being developed, which combine sufficient energy confinement to achieve fusion power amplification factors of $Q=10$, with benign heat loads to the plasma facing components. The radiative type-III ELMy 
H-mode seems a possible solution for such an integrated ITER scenario. Most notably the transient heat loads due to type-III ELMs are acceptable with even the most stringent boundary conditions.

This was achieved in experiments carried out with nitrogen seeding to mitigate the transient and steady-state heat flux to the divertor. The nitrogen seeding leads to an increased divertor and scrape-off-layer (SOL) radiation. This reduces the divertor electron temperature and the upstream SOL electron temperature. The divertor detaches in-between ELMs and nitrogen can penetrate further into the $\mathrm{X}$-point region affecting with its radiation also the gradient region of the pedestal. The pedestal temperature and consequentially the pedestal pressure are reduced. Simultaneously the pedestal width is increased. Eventually, with increased X-point radiation the plasma makes the transition from a type-I ELMy H-mode to a type-III ELMy H-mode. It was found experimentally that in type-III ELMy H-modes the pedestal width is larger than in type-I ELMy H-modes although the pedestal electron temperature and the pedestal pressure is reduced [6]. In the process of increasing the plasma radiation by nitrogen the ELM frequency is continuously increased and hence the energy loss per ELM is decreased leading to a reduction in the transient heat load to the plasma facing components. Additional radiative dissipation of the ELM energy is observed only for very small ELMs [7] but is not expected for larger ELMs as they will appear in ITER [8].

The reduction in the pedestal pressure by nitrogen seeding reduces the overall confinement by $\approx 8-20 \%$ as compared with the type-I ELMy H-mode baseline scenario. This reduction in stored energy can be regained by either (a) increasing the plasma current or (b) increasing the plasma core confinement. Both routes have been investigated at JET with the standard ELMy H-mode and the so-called hybrid scenario.

The experiments at JET were performed with nitrogen to mimic the ITER conditions with a medium- $Z$ impurity such as neon or argon, which radiate at higher temperatures as they will appear in ITER. In ITER the pedestal temperature is expected to be approximately twice as high as in JET. At JET impurities such as neon and argon do radiate further inside the core plasma [9] reducing the power flux across the pedestal top. This in turn reduces the ELM frequency [10]. Hence, with increasing radiative power fraction, by seeding argon for example, the ELM frequency is decreased leading to larger ELM energy losses from the plasma. This means essentially that impurities which radiate too far in the plasma core are not suited to reduce the transient heat loads to the plasma facing components without any additional means. In the following all experiments reported here refer to nitrogen seeded discharges.

\section{Highly radiative scenarios with type-III ELMy edge}

\subsection{Standard highly radiative nitrogen seeded ELMy H-mode with type-III ELMs}

Increasing the plasma current to $I_{\mathrm{p}}=17 \mathrm{MA}$ on ITER and hence reducing the edge safety factor $q_{95}$ to 2.6 would allow $Q=10$ operation at a reduced confinement enhancement factor of $H_{98(y, 2)}=0.75$ [11] at a high density of $100 \%$ the Greenwald density [12] $\left(\bar{n}_{\mathrm{e}} / n^{\mathrm{GW}}=N^{\mathrm{GW}}=1\right)$. Here $H_{98(y, 2)}$ is the ratio of energy confinement time normalized to the scaling of the energy confinement time $\tau_{\mathrm{E}} / \tau_{\operatorname{IPB} 98(y, 2)}$ resulting from a multi-device database [13]. The target values for this ITER operation scenario with a thermal stored energy of $W_{\text {th }}=325 \mathrm{MJ}$ are a normalized collisionality $v^{*}=0.042$, a normalized gyroradius $\rho^{*}=0.0015$, a normalized electron density $N^{\mathrm{GW}}=1$, an effective charge $Z_{\text {eff }} \leqslant 1.7$, a central line-averaged electron density $\bar{n}_{\mathrm{e}}=13.4 \times 10^{19} \mathrm{~m}^{-3}$, a pedestal energy $W_{\text {ped }}=0.35 \times W_{\text {th }} \approx 110 \mathrm{MJ}$, pedestal temperature $T_{\text {ped }}=2 \mathrm{keV}$ and a radiative power fraction $f_{\text {rad }}=0.75$. Here the dimensionless variables collisionality $v^{*}$ and ion gyroradius $\rho^{*}$ are calculated according to $v^{*}=$ $7.839 \times 10^{-4} \cdot q_{95} \cdot R_{0} \cdot\left(R_{0} / a_{\min }\right)^{1.5} \cdot\langle n\rangle \cdot Z_{\mathrm{eff}} \cdot\langle T\rangle^{-2}$ and $\rho^{*}=0.00457 \cdot \sqrt{m \cdot\langle T\rangle} \times\left(a_{\min } \cdot B_{0}\right)^{-1}$, where $\langle n\rangle$ and $\langle T\rangle$ are volume averaged values of density and temperature and $R_{0}$ and $a_{\min }$ are the major and minor radius, respectively. The effective ion charge of the plasma $Z_{\text {eff }}$ [14] is a measure of the purity of the plasma and has to be below 1.4 without the fusion ash helium and with the helium below 1.7.

This operation scenario was demonstrated at JET in a standard inductive scenario. To obtain these high densities high triangularity plasma configurations had to be chosen. All normalized parameters, including the ratio of the energy confinement time to the ITER confinement time scaling for ELMy H-mode operation $H_{98(y, 2)}$ (for the definition see, for example, [11]), the normalized electron density $N^{\mathrm{GW}}$, the normalized plasma pressure $\beta_{\mathrm{N}}$, the radiative power fraction $f_{\text {rad }}$, the plasma effective charge $Z_{\text {eff }}$ were met simultaneously [15]. In recent JET campaigns this plasma regime has been extended to plasma currents of $3.25 \mathrm{MA} / 2.6 \mathrm{~T}\left(q_{95}=2.6\right)$. In those high current discharges central line-averaged densities of up to $12 \times 10^{19} \mathrm{~m}^{-3}$ were reached. Figure 1 shows an overview of such a discharge. The plasma is heated mainly with neutral beam injection (NBI). Some ion cyclotron resonance heating (ICRH), up to $3 \mathrm{MW}$ in dipole polarization is added mainly to avoid impurity accumulation in the plasma centre. The thermal normalized pressure $\left(\beta_{\mathrm{N}}\right)$ reaches 1.6 in this discharge. The radiative power fraction is $70 \%$ and the ELM frequency is increased to almost $1 \mathrm{kHz}$. All pulses in the standard type-III ELMy $\mathrm{H}$-mode need a gas fuelling of $(0.5-1.0) \times 10^{23}$ electrons $\mathrm{s}^{-1}$, both for deuterium and for nitrogen.

\subsection{Hybrid scenario with nitrogen seeding and type-III ELMs}

An integrated hybrid type-III ELM regime with $\beta_{\mathrm{N}}^{\text {therm }}=2.2$ $\left(P_{\mathrm{NBI}} \approx 20-22 \mathrm{MW}\right)$ and $H_{98(y, 2)} \approx 0.83$ has been successfully developed on JET with nitrogen seeding (see figure 2). The target plasma scenario is a hybrid H-mode [16] (defined here as an optimized scenario for high $\beta_{\mathrm{N}}$ operation with moderate MHD activity) with type-I ELMs (\#68505), where pedestal plasma temperature is $T_{\text {ped }}=1 \mathrm{keV}$, $I_{\mathrm{p}}=1.7 \mathrm{MA}$, toroidal magnetic field $B_{\mathrm{t}}=1.7 \mathrm{~T}, \bar{n}_{\mathrm{e}} \approx 5 \times$ $10^{19} \mathrm{~m}^{-3}$ (70\% of the Greenwald density), $q_{95} \approx 3.2$ in which injected neutral beam power (NBI) is feed-back controlled to $\approx 14-16 \mathrm{MW}$ to achieve a $\beta_{\mathrm{N}}$ of 3 (non-thermal $\beta_{\mathrm{N}}$ ). The thermal confinement enhancement factor achieved in the target plasma scenario is $H_{98(y, 2)} \approx 1.05$ and the plasma effective charge is $Z_{\text {eff }}=1.8$. A high triangularity magnetic configuration $(\delta=0.44)$ is used. Lower hybrid heating is 


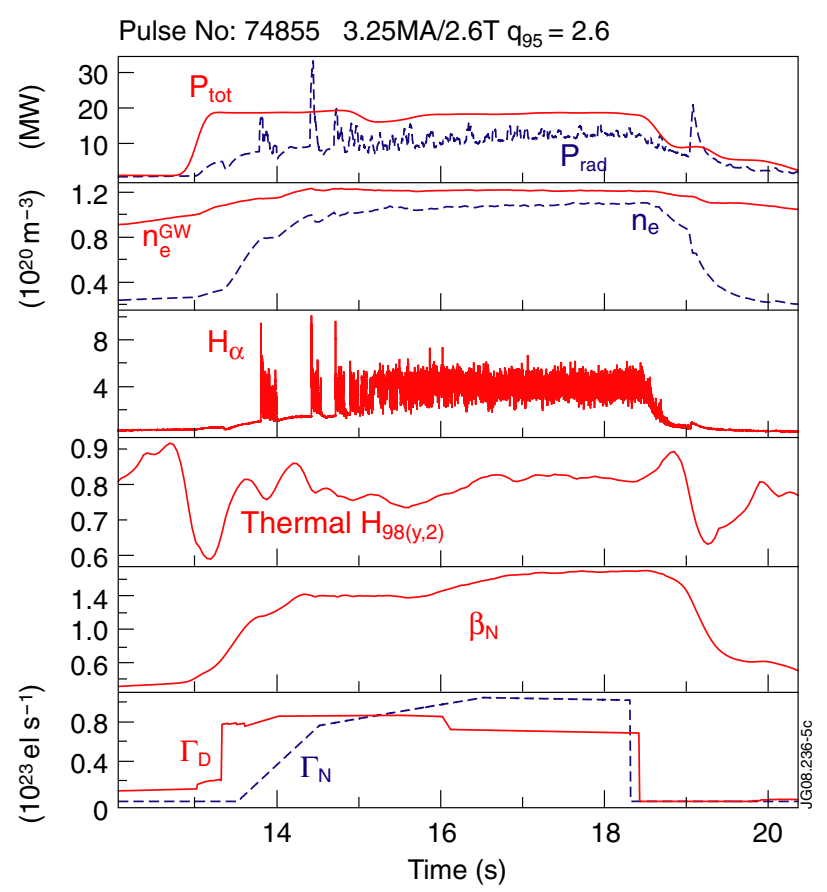

Figure 1. Overview of 3.25 MA/2.6 T type-III ELMy H-modes at JET (\#74855): total heating power $P_{\text {tot }}$; total radiated power $P_{\text {rad }}$; line-averaged density $\bar{n}_{\mathrm{e}}$, Greenwald density $n_{\mathrm{e}}^{\mathrm{GW}} ; H_{\alpha}$ emission in divertor; confinement enhancement factor $H_{98(y, 2)}$; normalized plasma pressure $\beta_{\mathrm{N}}$; deuterium fuelling in inner divertor $\Gamma_{\mathrm{D}}$; nitrogen fuelling in outer divertor $\Gamma_{\mathrm{N}}$.

used during the plasma current ramp up (for a duration of about $3 \mathrm{~s}$ ) to delay the plasma current profile penetration with the aim of producing a broad $q$-profile when the main heating is applied. This is followed by an intermediate $\beta_{\mathrm{N}}=2$ phase (for a duration of $3 \mathrm{~s}$ ) for stabilization of the $q$-profile close to 1 in order to minimize the impact of sawtooth on stability. The $\beta_{\mathrm{N}}$ request is then increased and kept constant for $4 \mathrm{~s}$.

During the main heating and high pressure phase, a preset injection of deuterium is applied: $\beta_{\mathrm{N}}=3$ has been obtained with low deuterium fuelling (\#68505: $\Gamma_{\mathrm{D}}=0.6 \times$

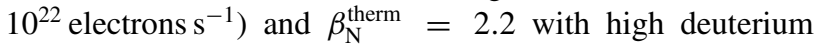
fuelling and density close to the Greenwald density $\bar{n}_{\mathrm{e}}=$

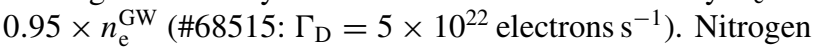
injection is applied in addition to deuterium fuelling during the first three seconds of the high $\beta_{\mathrm{N}}$ plateau (when $\beta_{\mathrm{N}}^{\text {therm }}=2.2$ ). Deuterium is injected in the bottom of the divertor near the outer strike point on the low field side (LFS) while nitrogen is injected into the private-flux region from the horizontal target plate located on the high field side (HFS). The maximum radiated power fraction achieved with deuterium fuelling alone (with mainly $\mathrm{D}$ and $\mathrm{C}$ radiators) is $P_{\mathrm{rad}} / P_{\text {heat }}=0.45$ with density close to the Greenwald limit $\bar{n}_{\mathrm{e}} \approx n^{\mathrm{GW}}$ (\#68739). Using deuterium plus nitrogen fuelling enables one to increase the radiative power fraction. The transition to the type-III ELM regime is observed at $P_{\text {rad }} / P_{\text {heat }} \geqslant 0.55$. A maximum of $70 \%$ radiative power fraction has been achieved during this experiment. In those discharges nitrogen contributes similar to the radiative power fraction as carbon. In the type-III ELM phase the pedestal ion temperature $T_{\text {ped }}$ is reduced to values below $750 \mathrm{eV}$. The degradation of global confinement associated with the type-III ELM regime is

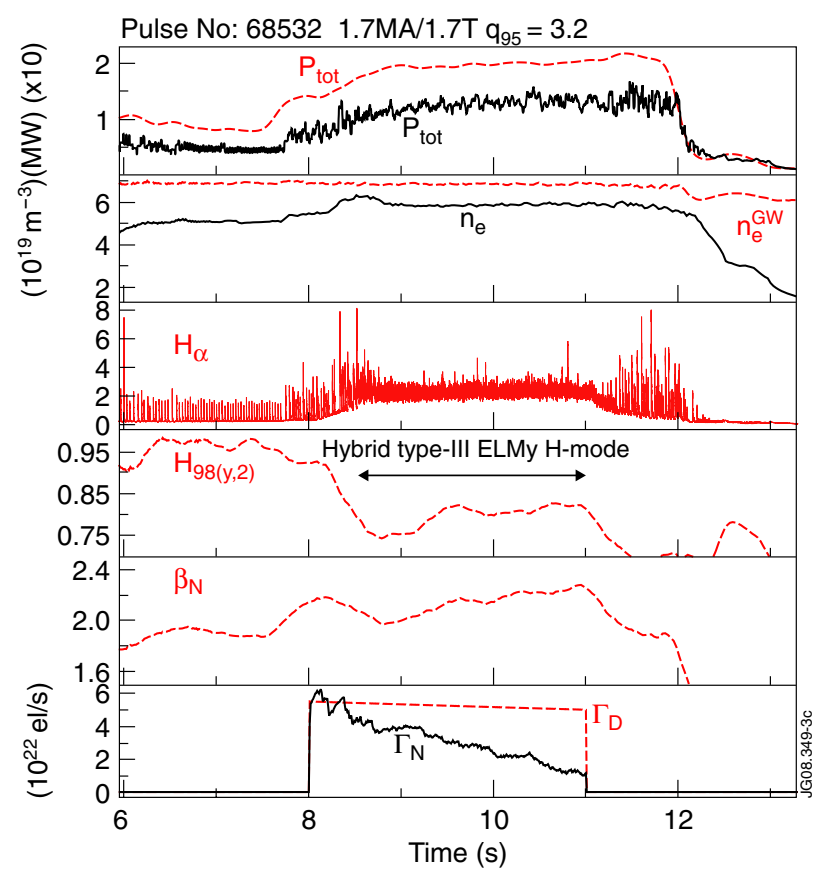

Figure 2. Overview of a 1.7 MA/1.7 T type-III Hybrid discharge at JET (\#68515): total heating power $P_{\text {tot }}$; total radiated power $P_{\text {rad }}$; line-averaged density $\bar{n}_{\mathrm{e}}$, Greenwald density $n_{\mathrm{e}}^{\mathrm{GW}} ; \mathrm{H}_{\alpha}$ emission in divertor; confinement enhancement factor $H_{98(y, 2)}$; normalized plasma pressure $\beta_{\mathrm{N}}$; deuterium fuelling in inner divertor $\Gamma_{\mathrm{D}}$; nitrogen fuelling in outer divertor $\Gamma_{\mathrm{N}}$.

about $10 \%$ compared with the reference hybrid high Dfuelling discharge (\#68515) at similar deuterium fuelling rates of $\Gamma_{\mathrm{D}}=5 \times 10^{22}$ electrons s $^{-1}$.

In those discharges it was not possible to increase the plasma core performance to compensate for the energy loss at the pedestal. The overall confinement of the type-III ELM hybrid scenario is very similar to the confinement of the standard type-III ELMy H-mode.

\section{Power load to the plasma facing components}

It has been found that the power load to the divertor can be reduced significantly in the nitrogen seeded type-III ELMy $\mathrm{H}$-mode. In the steady state a radiative power fraction of $97 \%$ was already achieved [15]. Most of the power (75\%) is radiated in the divertor and $\mathrm{X}$-point region outside a minor radius of $\rho=r / a_{\min } \leqslant 0.9$, hence just on top and outside the pedestal. The convective power to the divertor target is minimized and the divertor targets only experience power fluxes from the $\mathrm{X}$-point radiation.

More importantly, the transient heat loads due to ELMs can be reduced significantly in the radiative cooled type-III ELMy H-mode [7,8]. This is mainly because of the reduced ELM energy loss from plasma in type-III ELMy H-modes. Radiative dissipation of the ELM power fluxes from the pedestal to the divertor is only marginal for type-I ELMs [8, 17] and is only observed for very small ELMs in type-III ELMy H-modes [7]. For instance, on JET the transient heat loads due to type-III ELMs onto the outer divertor target were reduced to $2 \mathrm{~kJ} \mathrm{~m}^{-2}$ [7]. With the new infrared diagnostic capabilities [18] better measurements with improved time resolution are 


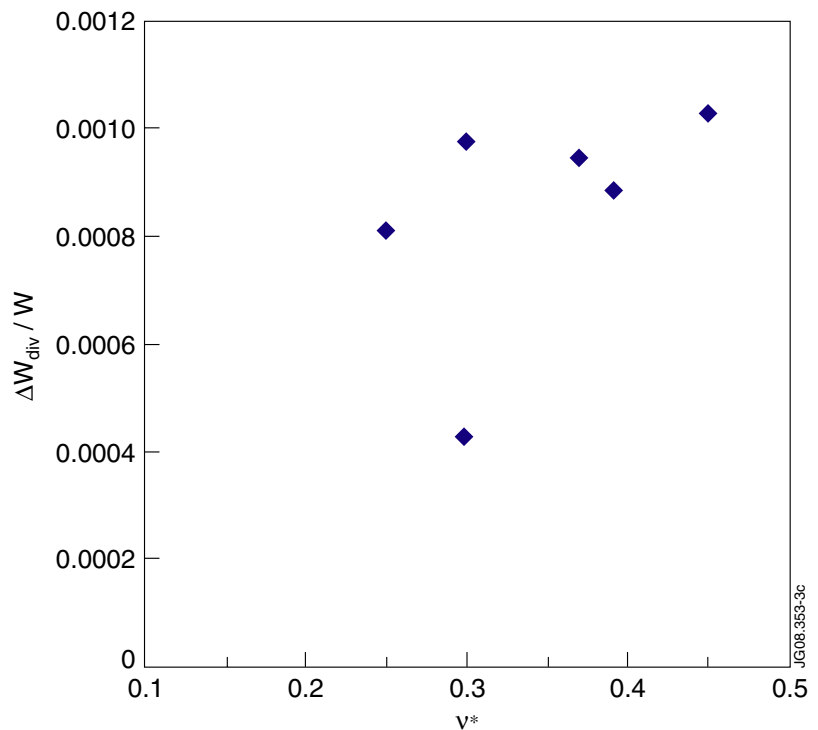

Figure 3. $\Delta W_{\text {div }}^{\mathrm{ELM}} / W$ as a function of collisionality as derived from IR thermography for 2.5 MA/2.0 T type-III ELMy H-modes at JET.

possible. For the highly radiative nitrogen seeded plasmas the temperature excursions in the outer divertor due to the type-III ELMs is only in the range of $10^{\circ} \mathrm{C}$. This corresponds to a power flux density of about $1 \mathrm{MW} \mathrm{m}^{-2}$. At the inner divertor it is typically half of that value. No difference between the standard type-III ELMy H-mode and the hybrid type-III ELMy H-mode was observed. The divertor heat load due to the type-III ELMs normalized to the total stored energy versus the core collisionality is shown in figure 3 . The data set shows no apparent dependence of the ELM energy deposition in the divertor as a function of collisionality. If at all, the ELM energy loss is slightly decreasing with decreasing collisionality. This is different from the radiative type-I ELMy $\mathrm{H}$-modes, where the energy loss per ELM is increasing towards lower collisionalities [17]. The absolute value of the type-III ELM energy deposition is about $0.1 \%$. Taking a constant ratio between the ELM energy deposited on the divertor target $\left(\Delta W_{\text {div }}^{\mathrm{ELM}}\right)$ and the total stored energy $(W) \Delta W_{\text {div }}^{\mathrm{ELM}} / W \approx 0.1 \%$ for type-III ELMs in a radiating scenario the ITER divertor load would be about $0.3 \mathrm{MJ}$. This would approximately translate into $0.1 \mathrm{MJ} \mathrm{m}^{-2}$.

However, if a collisionality dependence of $\Delta W_{\mathrm{div}}^{\mathrm{ELM}} / W$ similar to type-I ELMs is assumed, then the energy load to the divertor could be a factor of 3 higher, leading to a predicted energy load of $0.3 \mathrm{MJ} \mathrm{m}^{-2}$. It appears that the ELM rise time for type-III ELMs is much slower than for type-I ELMs. Details of the temporal evolution and spatial energy distribution could influence the predictions and need to be further investigated. Even more difficult to predict is the ELM frequency of the radiative type-III ELMs. At JET the ELM frequencies vary between $150 \mathrm{~Hz}$ and $1 \mathrm{kHz}$.

\section{Confinement scaling}

The main drawback of the type-III ELMy H-mode is its reduced confinement, when compared with the type-I ELMy H-mode. The JET steady-state database contains about 576



Figure 4. $H_{98(y, 2)}$ as a function of the radiative power fraction for type-III ELMy H-modes with nitrogen seeding.

type-III ELMy H-modes and 672 type-I ELMy H-modes. The fit gives a $H_{98(y, 2)}$ of 0.95 for the type-I ELMy H-modes and a $H_{98(y, 2)}$ of 0.87 for the type-III ELMy H-modes [19]. The parametric dependence in the scaling is otherwise the same for type-I ELMy H-modes and type-III ELMy H-modes. However, for strongly radiating type-III ELMy H-modes the confinement appears to be slightly lower. To investigate this further, a large database of highly radiative type-III ELMy $\mathrm{H}$-modes on JET has been set up, including discharges in low triangularity (lower density) [20] and high triangularity (higher density) magnetic configurations. The more recent data added to that database are from discharges at high density, low edge safety factor and high triangularity. The high triangularity allows to operate at higher density without sacrifice in confinement. This has been first shown in deuterium seeded type-I ELMy H-modes [21, 22] and impurity seeded type-I ELMy H-modes [23]. This results in an increase in the product $N^{\mathrm{GW}} \times H_{98(y, 2)}$ by approximately $20 \%$. No principal deterioration of the confinement towards very high radiative power fractions was found. The confinement is similar in very strongly radiating plasmas with radiative power fractions close to $100 \%$ and in plasmas close to the type-I to type-III ELMy $\mathrm{H}$-mode transition at $64 \%$ radiative power fraction (see figure 4).

For extrapolations to ITER it is important to compare the experimental results with the scaling laws used to design ITER. We will compare here our experimental results with the confinement time scaling for ELMy H-modes $\tau_{\operatorname{IPB} 98(y, 2)} \propto$ $\tau_{\mathrm{B}} \rho^{*-0.7} \beta^{-0.9} v^{*-0.01}$ with $\tau_{\mathrm{B}}$ being the Bohm confinement time $\tau_{\mathrm{B}} \propto a_{\min } B_{\mathrm{t}} / T$. Written without the Bohm confinement time the relation between the scaled energy confinement time and the dimensionless parameters is $B_{\mathrm{t}} \times \tau_{\operatorname{IPB} 98(y, 2)} \propto$ $\rho^{*-2.7} \beta^{-0.9} v^{*-0.01}$. In particular, the variation in collisionality $\nu^{*}$ and normalized gyroradius $\rho^{*}$ are important.

Figure 5 shows the confinement enhancement factor $H_{98(y, 2)}$ as a function of the collisionality. Table 1 shows the large operational range covered by this database. Radiative 


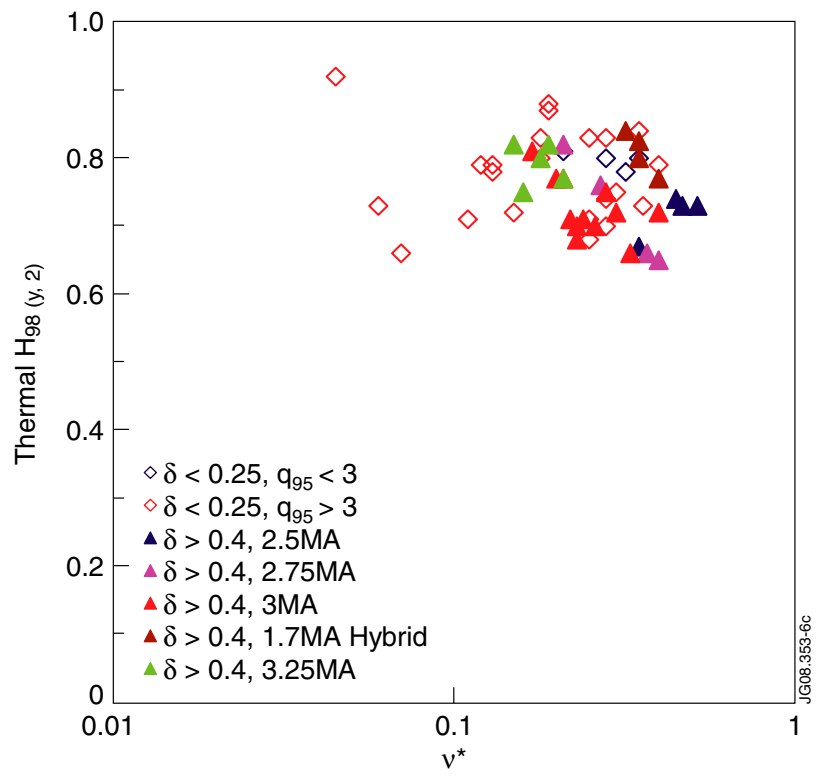

Figure 5. $H_{98(y, 2)}$ as a function of collisionality for radiative type-III ELMy H-modes at JET.

Table 1. Operational range of type-III ELMy H-modes with nitrogen seeding.

\begin{tabular}{ll}
\hline Parameter & Range \\
\hline$I_{\mathrm{p}}$ & $0.95-3.25 \mathrm{MA}$ \\
$B_{\mathrm{t}}$ & $1.0-3.45 \mathrm{~T}$ \\
$q_{95}$ & $2.3-4.3$ \\
$\delta$ & $0.18-0.47$ \\
$N_{\mathrm{GW}}$ & $0.4-1.0$ \\
$\beta_{\mathrm{N}}$ & $0.9-2.6$ \\
$P_{\text {heat }}$ & $2.3-33 \mathrm{MW}$ \\
$P_{\text {heat }} / P_{\mathrm{LH}}$ & $1.0-3.4$ \\
\hline
\end{tabular}

type-III ELMy H-modes are obtained at radiative power fractions larger than 0.55 . It also includes hybrid discharges. The operational domain covered at JET does extend from $0.95 \mathrm{MA} / 1 \mathrm{~T}$ AUG, CDH-mode [24] identity pulses up to $3.25 \mathrm{MA}$ with high and low $q_{95}$, as well as $2.5 \mathrm{MA} / 3.45 \mathrm{~T}$ low $\rho^{*}$ pulses with heating powers of up to $33 \mathrm{MW}$. The power over the $\mathrm{L}-\mathrm{H}$ mode power threshold, as defined, $P_{\mathrm{LH}}=$ $0.042 n_{\mathrm{e}}^{0.73} B_{\mathrm{t}}^{0.74} S^{0.98}$ (in MW, $10^{20} \mathrm{~m}^{-3}, \mathrm{~T}$ and $\mathrm{m}^{-3}$ ) [13], varies between 1.0 and 3.4. No systematic difference between standard ELMy H-modes and hybrid discharges was observed. The data set shows no apparent dependence of the confinement enhancement factor on collisionality. However, looking only at the high triangularity discharges at low edge safety factor leads to the suggestion that the confinement is slightly increasing towards lower collisionality. In figure 6 the confinement at collisionalities close to 0.1 is about $H_{98(y, 2)} \approx 0.85$ in $3.25 \mathrm{MA}$ discharges.

In figure 7 the $H_{98(y, 2)}$ is shown versus the normalized gyroradius. Within one plasma current, magnetic field and configuration the $H_{98(y, 2)}$ does decrease with decreasing $\rho^{*}$. This is an effect of the increased gas fuelling in those experiments, which leads to lower pedestal as well as core temperatures. However, all those lines are parallel to each other. Comparing the data at high $\rho^{*}$ with the $2.5 \mathrm{MA} / 3.45 \mathrm{~T}$ pulses at low $\rho^{*}$ shows an increase in the $H_{98(y, 2)}$ with

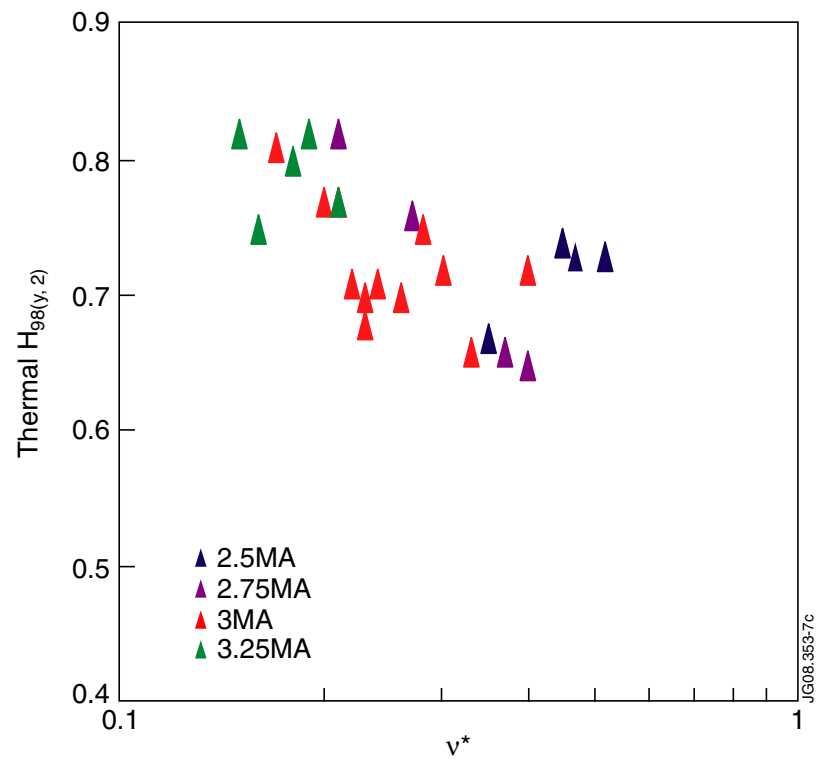

Figure 6. $H_{98(y, 2)}$ as a function of collisionality for radiative type-III ELMy H-modes in high triangularity configurations $\delta=0.44$ and low $q_{95}=2.6$ scenario at JET

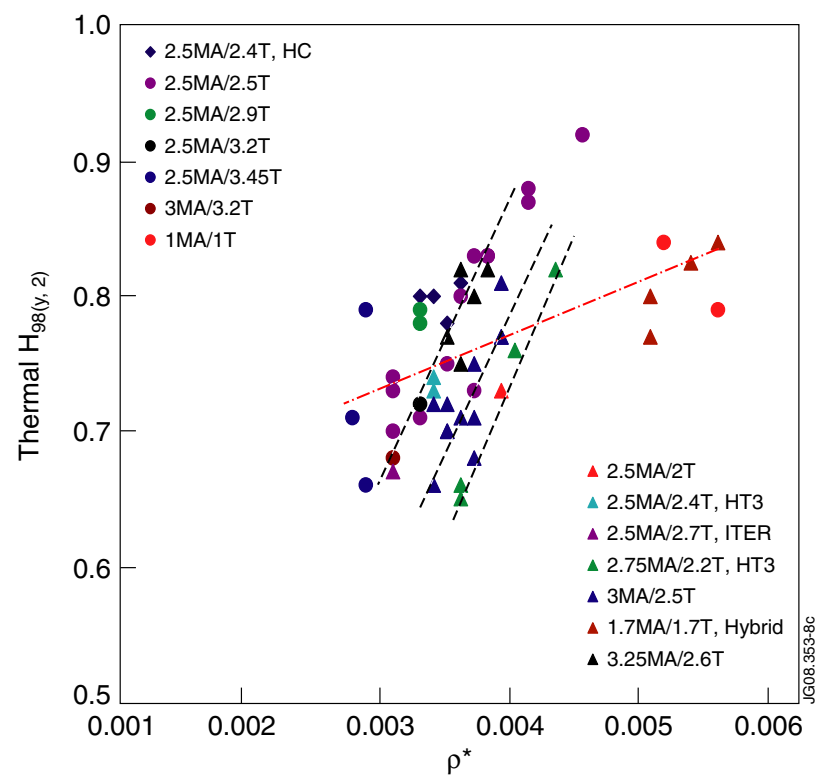

Figure 7. $H_{98(y, 2)}$ as a function of normalized gyroradius $\rho^{*}$ for radiative type-III ELMy $\mathrm{H}$-modes at JET: the dashed lines guide the eye, the dashed-dot line reflects the scaling $\rho^{*-2.5}$.

$\rho^{*}$ roughly proportional to $\rho^{* 0.2}$. This suggests that the confinement time scaling $\tau_{\operatorname{IPB} 98(y, 2)}$ scales stronger with $\rho^{*}$ than its actual scaling, leading to $B_{\mathrm{t}} \times \tau_{\mathrm{IPB} 98(y, 2)} \propto$ $\rho^{*-2.5}$. However this could be due to a different $\beta$ dependence in the $H_{98(y, 2)}$ scaling [25]. Neglecting this would lead to a scaling which is closer to gyro-Bohm scaling: $B_{\mathrm{t}} \times \tau_{\text {E-gyro-Bohm }} \propto \rho^{*-3.0}$.

\section{Edge operational space}

Figure 8 shows the edge operational space of the high triangularity discharges with nitrogen seeding. The 


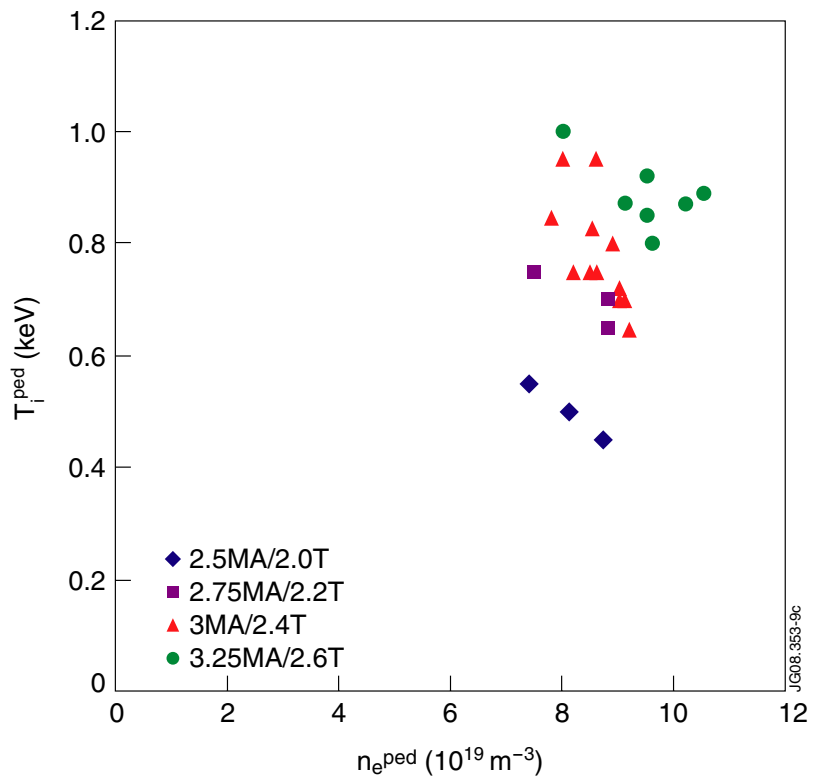

Figure 8. Pedestal ion temperature as a function of the pedestal electron density for radiative type-III ELMy H-modes at JET.

ion pedestal temperature is taken from charge exchange spectroscopy and the pedestal electron density is taken from LIDAR. Figure 8 shows data for discharges from $2.5 \mathrm{MA} / 2.0 \mathrm{~T}$ to $3.25 \mathrm{MA} / 2.6 \mathrm{~T}$ at a constant edge safety factor of $q_{95}=2.6$. Remarkable is the strong increase in the electron temperature. By lowering the collisionality the pedestal temperature was increased from $0.4 \mathrm{keV}$ to about $1.0 \mathrm{keV}$ in the high triangularity discharges (see figure 9). This has to be extrapolated to the 17 MA ITER $Q=10$ scenario at $N^{\mathrm{GW}}=1$, for which a pedestal temperature of $2-2.5 \mathrm{keV}$ is necessary $[26,27]$. In figure 9 the ITER value is shown together with the JET pedestal temperature data illustrating the gap in the data. Although global data for much lower collisionalities for nitrogen seeded type-III ELMy $\mathrm{H}$-modes are available, those pulses lack good pedestal data and cannot be used for the extrapolations. The model proposed by Pogutse and Igitkhanov [26,27] described reasonably well the edge operational space of unfuelled type-III ELMy H-mode at low collisionality [28]. In this model the type-III ELMs are described by an instability based on resistive interchange modes driven by magnetic flutter (RIF). When the radial electric field just inside the separatrix becomes sufficiently strong, it can stabilize the RIF [28]. The model gives a critical pedestal temperature for the transition from type-III ELMs to type-I ELMs, which is different for high and low collisionalities. This means all type-III ELMy $\mathrm{H}$-mode pedestal temperatures should be below this critical temperature. For high collisionality the critical temperature is only weakly proportional to the magnetic field $\left(T_{0 \text { crit }} \propto\right.$ $q_{95}^{24 / 17} B_{\mathrm{t}}^{10 / 17}$ ), whereas for low collisionality the dependence on the magnetic field is stronger $\left(T_{0 \text { crit }} \propto q_{95}^{18 / 5} B_{\mathrm{t}}^{2}\right)$. The data of the nitrogen seeded type-III ELMy H-modes shown in figure 8 demonstrate that the pedestal temperature can be increased by about a factor of 2 when increasing the magnetic field from the 2 to $2.6 \mathrm{~T}$. This leads to the conclusion that the dependence on the magnetic field is closer to $B_{\mathrm{t}}^{2}$ scaling or even stronger. As

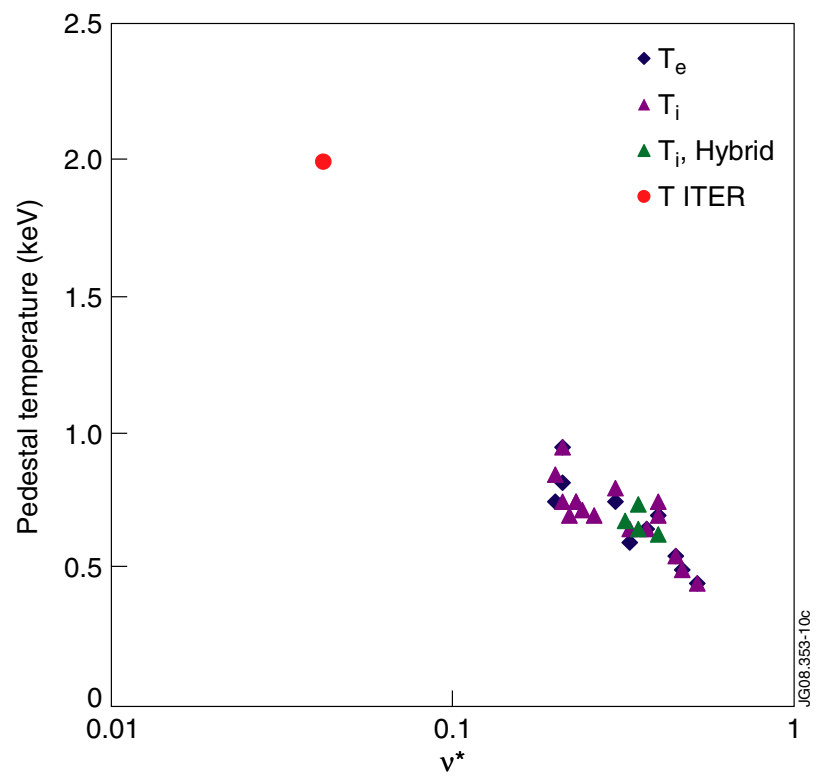

Figure 9. Pedestal temperature as a function of collisionality for radiative type-III ELMy $\mathrm{H}$-modes at JET.

figure 9 shows, the collisionality varies by more than a factor of 3 from high collisionality to intermediate collisionality. This could be an explanation why the critical temperature is increasing so strongly with the magnetic field. And this could also explain why the confinement enhancement factor $H_{98(y, 2)}$ is slightly increasing towards lower collisionality as figure 6 suggests. However, the model of Pogutse and Igitkhanov does not reflect the $q_{95}$ dependence of the experiments [28]. Hence some uncertainties in the prediction of the type-I to type-III ELMy H-mode threshold remain.

Often type-III ELMs are also associated with resistive ballooning instabilities. A model based on resistive ballooning instabilities was used to describe the type-I to type-III ELM back transition in strongly fuelled discharges [29]. Hence this model is based on the dimensionless pressure gradient and the collisionality. A critical density for the back transition to type-III ELMs can be derived: $n_{\mathrm{e}, \text { crit }} \propto$ $B_{\mathrm{t}} \sqrt{f(s)} /\left(q_{95}^{5 / 4} R^{3 / 4} Z_{\text {eff }}^{1 / 4}\right)$, with $f(s)$ being a function of the shear. This model reflects better the inverse $q_{95}$ dependence on the back transition as found in $\mathrm{H}$-mode density limit experiments [28]. Both the inverse $q_{95}$ dependence and the inverse $Z_{\text {eff }}$ dependence will be favourable for the proposed ITER scenario at $17 \mathrm{MA}$ and $N^{\mathrm{GW}}=1$. In summary, based on the model of Chankin and Saibene, high pedestal densities should be possible in this regime.

The evidence shown above, that at lower collisionalities the dependence of the critical temperature for the type-III to type-I ELM transition on the magnetic field is strong, leads to the suggestion that for ITER a higher pedestal temperature can be expected and hence higher pedestal pressure. A higher pedestal temperature would also result in a higher plasma core temperature assuming stiff temperature profiles. Altogether, this might increase the fusion power performance of this proposed type-III ELMy H-mode ITER scenario. 
- D and $\mathrm{N}$ fuelled, $\delta>0.4$

ITER, 17MA, $\mathrm{H}_{98}=0.75$

- $\mathrm{D}$ and $\mathrm{N}$ fuelled, $\delta<0.25, \mathrm{q}_{95}<3$

口 Unfuelled

- D and $\mathrm{N}$ fuelled, $\delta<0.25, \mathrm{q}_{95}>3$

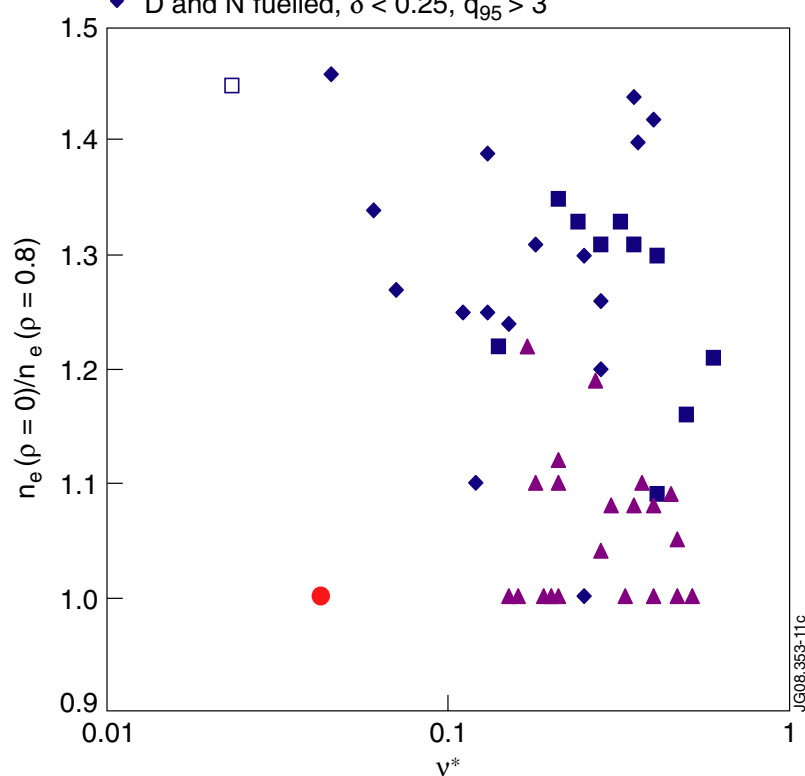

Figure 10. Peaking of the electron density profile versus the collisionality for radiative type-III ELMy H-modes at JET.

\section{Profile peaking}

All ITER simulations are based on a flat density profile. The confinement could be improved, if the plasma core density could be increased above the value of the pedestal density. It is suggested that towards lower plasma collisionality the density is peaked in the plasma centre [30]. The experiments described in [30] are type-III ELMy H-modes without any additional gas fuelling, just fuelled by NBI. The strongly radiating type-III ELMy H-modes presented here have strong external gas fuelling by deuterium and nitrogen. Figures 10 and 11 show the electron density peaking as derived from LIDAR versus the collisionality and versus the normalized electron density. It is usually difficult to separate the effect of density from the effect of the collisionality due to its co-linear behaviour [31]. However, careful analysis suggests the presence of an anomalous pinch leading to density peaking, which could increase the fusion power in ITER by almost $30 \%$ [31]. Figure 10 suggests a density peaking with decreasing collisionality; however figure 11 suggests that the density peaking is more related to the normalized density (and maybe underlying effects of core fuelling versus edge fuelling). A detailed transport study, including the effect of neutral beam fuelling as well as gas fuelling at the plasma edge, has not been carried out. However, it seems that the correlation between density peaking and normalized density is larger than the one with density peaking and collisionality. Explicitly the regressions yield $n_{\mathrm{e}}(0) / n_{\mathrm{e}}(0.8)=1.0784-0.0758 \ln v^{*}$ with $R^{2}=0.115$ and $n_{\mathrm{e}}(0) / n_{\mathrm{e}}(0.8)=1.5935-0.5287 N^{\mathrm{GW}}$ with $R^{2}=0.4478$. The stronger correlation with the normalized density can result from the strong gas fuelling in those type-III ELMy H-mode discharges. Investigations on the density peaking are usually restricted to type-I ELMy

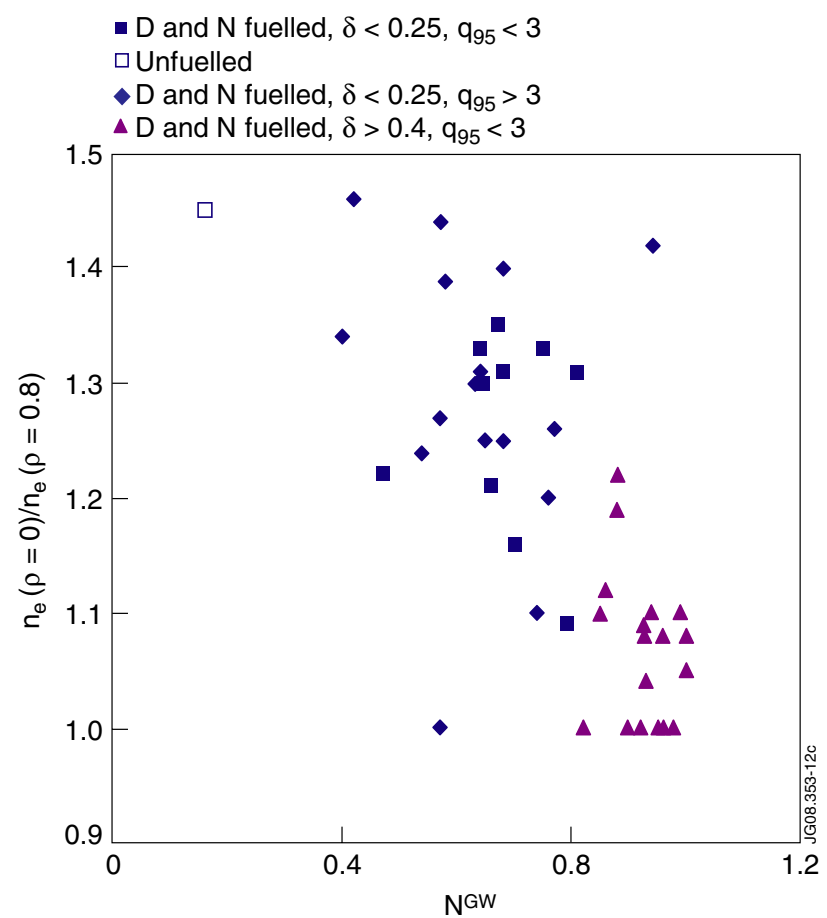

Figure 11. Peaking of the electron density profile versus the normalized electron density $N^{\mathrm{GW}}=\bar{n}_{\mathrm{e}} / n^{\mathrm{GW}}$ for radiative type-III ELMy H-modes at JET.

$\mathrm{H}$-modes without gas fuelling [31] and even the neutral beam fuelling is reduced to avoid any ambiguity in the determination of the inward pinch velocity resulting from particle sources [30]. However, the plasma core in type-I ELMy H-modes and type-III ELMy H-modes should behave similarly. Nongas fuelled type-III ELMy H-modes experience similar density peaking as type-I ELMy H-modes [30] as shown in figure 10 with the low collisionality unfuelled type-III ELMy H-mode.

It should be noted that density peaking not only is favourable for the fusion power production but might also lead to an increased impurity concentration in the plasma core. In particular, high- $Z$ elements tend to accumulate in the plasma centre as a result of neoclassical transport in the plasma core, when the density profile is peaked. This accumulation of high- $\mathrm{Z}$ elements might even be amplified in impurity seeded discharges [32] due to impurity-impurity driven forces. The loss of sawtooth activity then has a detrimental effect on the core plasma pollution.

\section{Plasma pollution}

The plasma pollution is a result of impurity production, plasma transport and radiation efficiency. Both impurity production and radiation efficiency are strongly dependent on the plasma density. Hence, the plasma pollution $\left(Z_{\text {eff }}\right)$ depends strongly on the absolute density in those highly radiating plasmas. In recent JET campaigns the standard radiating type-III ELMy $\mathrm{H}$-mode has been extended to higher plasma current (3.25 MA) and therefore high absolute density. At those high densities the $Z_{\text {eff }}$ is strongly reduced. The effective charge $Z_{\text {eff }}$ was reduced from 2.2 to values below 1.5 , mainly due to the increased absolute density and reduced carbon erosion. In those highly 
radiative discharges $\left(f_{\text {rad }} \geqslant 0.75\right)$ nitrogen, having replaced carbon, is the main radiator and the dominant impurity in the plasma.

The impurity production in the divertor is generally reduced towards higher density and higher radiative power fraction [15,33]. A comparison of the carbon erosion in the divertor and the main chamber wall revealed that the main chamber wall erosion is much lower in type-III ELMy $\mathrm{H}$ modes, when compared with type-I ELMy H-modes [33]. This is also reflected in the hydrogen retention in the type-III ELMy $\mathrm{H}$-modes, which is determined by the co-deposition of carbon and hydrogen in the inner divertor. The carbon transported to the inner divertor does have its origin mainly in the main chamber. The chemical erosion of carbon is enhanced by the chemical erosion through nitrogen. Detailed investigations are ongoing and will be reported in the future.

Furthermore, the divertor geometry and the fuelling location have an impact on the plasma pollution [34]. A closed divertor leads to a stronger detachment at similar electron densities when compared with an open divertor. Also fuelling close to the outer divertor strike point can reduce the plasma pollution [34]. However, full detachment can substantially increase the penetration depth of deuterium and impurity ions leading to a higher $Z_{\text {eff }}$ in the plasma core. This increased penetration depth is a general behaviour in impurity seeded discharges (see also [35]).

Naturally, for the type-III ELMy hybrid mode the $Z_{\text {eff }}$ is higher since the density is lower and the heating power is much higher. At a density of $\bar{n}_{\mathrm{e}}=7 \times 10^{19} \mathrm{~m}^{-3}$ the $Z_{\text {eff }}$ is about 3 .

On the basis of an enlarged database a new $Z_{\text {eff }}$ scaling has been developed [33]: $Z_{\text {eff }}=1+40 P_{\text {rad }} Z^{0.12} \tau_{\mathrm{E}} S^{-0.94} n_{\mathrm{e}}^{-1.5}$ $a_{\mathrm{min}}^{-1} R^{-1}$, with $S$ being the plasma surface, $a_{\min }$ the minor radius and $R$ the major radius. The main improvement of this scaling is the introduction of the impurity transport. This has already been done successfully for other tokamaks [36]. For the high density 17 MA scenario with a fusion power of $400 \mathrm{MW}$ and a $H_{98(y, 2)}=0.75$, at a density of $N^{\mathrm{GW}}=1$ a $Z_{\text {eff }}$ of 1.9 is predicted, excluding any contribution from helium. This is above the assumptions made for ITER, which include helium. However, details of the radial impurity transport and profile effects in the temperature and density profiles are not taken into account. For the standard $15 \mathrm{MA}$ ITER scenario with $H_{98(y, 2)}=1$ and a density of $N^{\mathrm{GW}}=0.85$ the $Z_{\text {eff }}=2.5$ is worse. A review of the tokamak size dependence is necessary, however, to reduce error bars in this prediction.

\section{MHD stability}

All ELMy H-modes at JET are marginally unstable to the development of neoclassical tearing modes (NTMs) [37]. The radiative scenarios are particularly prone to NTMs, because of the dependence of the marginal beta $\beta_{\text {Nmarg }}$ on the normalized poloidal ion gyroradius $\rho_{\mathrm{p} . \mathrm{i}}^{*}=\rho_{\mathrm{p}, \mathrm{i}} / a_{\min }$ [38], which is low in the radiative scenarios with the cooled pedestal. Seed islands produced by large sawtooth crashes can trigger the NTMs, when $\beta_{\mathrm{N}}$ is larger than the critical marginal $\beta_{\mathrm{Nmarg}}$ [37]. The disturbance and hence the seed island are particularly large in sawtooth crashes where a large volume is affected, hence large sawtooth inversion radius. Operation at $q_{95}=2.6$ has the disadvantage of having a large sawtooth inversion radius,

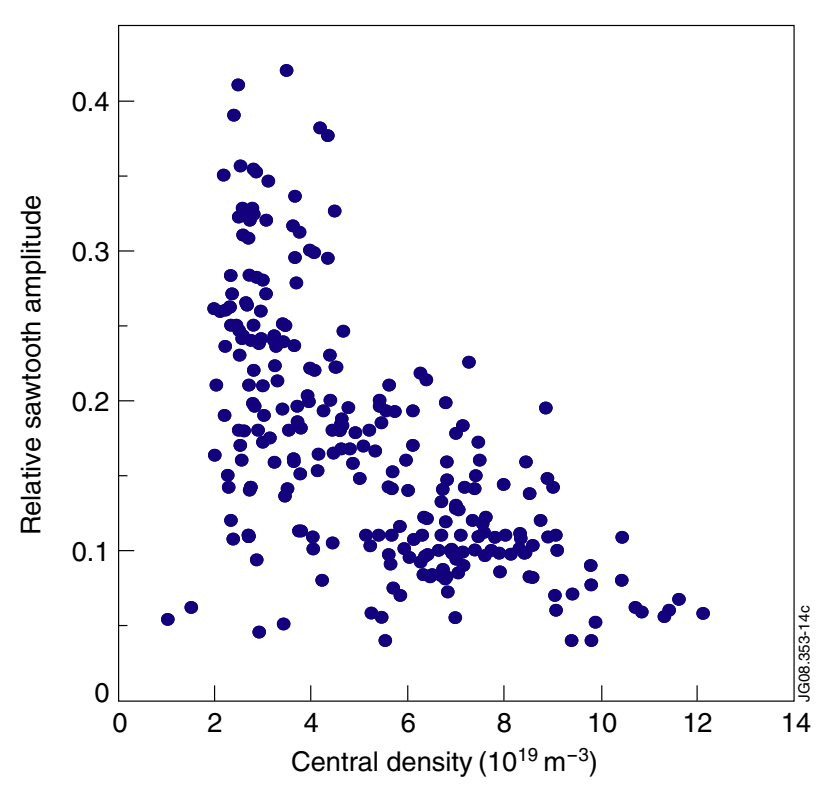

Figure 12. Relative sawtooth amplitude of the plasma pressure $\Delta p / p$ in the plasma centre versus the central density for JET NBI heated plasmas (with deuterium fuelling).

which potentially leads to larger sawtooth crashes. A scenario had to be developed to avoid triggering the NTMs $(m / n=3 / 2)$ in the transient period of the ramp up in the radiative scenario, where the sawtooth period is the longest. Here $m$ and $n$ are the poloidal and toroidal mode numbers, respectively. Setting $q_{95}=3.4$ at the beginning of the heating period and ramping down to $q_{95}=2.6$, once regular sawtooth activity is obtained, is the major recipe to avoid NTMs. During the flat top period of the highly radiative scenario, sawtooth activity is benign. In particular at the high plasma densities, which are an integral part of the highly radiative type-III ELMy $\mathrm{H}$-mode, the relative sawtooth amplitude of the plasma pressure $(\Delta p / p)$ is small (see figure 12). To prevent any impurity accumulation in the plasma core ICRH is added with a heating power of 1-3 MW. This is a typical method to maintain sawtooth activity in impurity seeded discharges [39].

However, large ELMs can influence the trigger of NTMs. A minimum gas fuelling is necessary throughout the heating power phase to avoid large ELMs and to keep the ELM frequency at a level above $20 \mathrm{~Hz}$. In figure 13 a comparison of two plasma pulses with different gas fuelling recipes is shown. In pulse \#74344 the gas fuelling of both deuterium and nitrogen is too low and hence the ELM frequency is below $20 \mathrm{~Hz}$. This triggers a $3 / 2 \mathrm{NTM}$ at $15.45 \mathrm{~s}$ as can be seen in detail in figure 14 by the growth of the $n=2$ activity. When the gas fuelling is increased by almost a factor of 2 the ELM frequency is increased sufficiently to avoid triggering of ELMs.

The MHD activity observed during the hybrid type-III ELM operation is characterized by mild $n=1$ sawteeth precursors present during the high $\beta_{\mathrm{N}}$ and $n=3$ mode presents near the $q=4 / 3$ surface [40]. The more deleterious $2 / 1$ and $3 / 2$ NTM are not present during the hybrid type-III ELMy case nor in the reference type-I case. The core $q$-profile of the integrated hybrid type-III ELM is very similar to the $q$-profile of the reference hybrid type-I ELMy scenario, indicating that type-III ELMy operation obtained with nitrogen injection is 


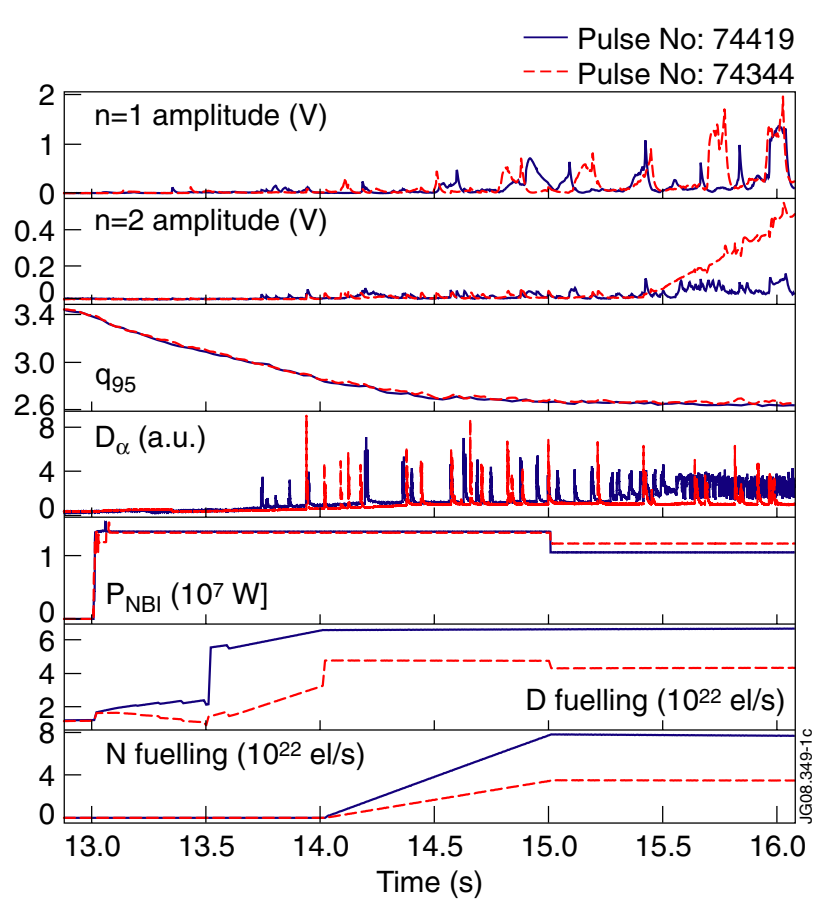

Figure 13. Comparison of two plasma pulses with different fuelling; $n=1$ MHD activity, $n=2$ MHD activity, $q_{95}, H_{\alpha}$ light in divertor, neutral beam power, deuterium gas fuelling rate, nitrogen gas fuelling rate: \#74344 develops a 3/2 NTM; \#74410 does not develop any NTM.

compatible with high $\beta_{\mathrm{N}}$ operation with minimum impact of sawteeth on the stability.

\section{Extrapolation to ITER with the integrated model COREDIV}

The nitrogen seeded high triangularity JET discharges have been modelled with COREDIV [41]. COREDIV is an integrated model solving self-consistently the $1 \mathrm{D}$ energy and particle transport of plasma and impurities in the core region and 2D multifluid transport in the SOL [42]. The energy confinement is scaled with the empirical $H_{98(y, 2)}$ scaling. The target erosion by nitrogen is not included. The physical carbon target erosion is dominated by deuterium and selfsputtering. The chemical erosion yield is calculated according to the flux dependence given in [43]. No main chamber erosion was included in the calculations. However, the experimental data (main plasma profiles in the core, the radiated power and the plasma pollution) were reconstructed satisfactorily. On the basis of this benchmarking to JET experiments predictions for ITER were done. In the ITER case neon was seeded as radiating impurity. The divertor target was a carbon target. Figures 15 and 16 show the results of the COREDIV simulations for the 15 MA standard ITER scenario for a set of densities and confinement enhancement factors. The results indicate, with reasonable accuracy, that this plasma scenario can achieve a power amplification factor $Q$ in excess of 6 at $15 \mathrm{MA}\left(q_{95}=3.0\right)$ with auxiliary heating powers of $40 \mathrm{MW}$. Higher heating power will have a detrimental effect on the fusion amplification factor. However, as reported above, a slight increase in confinement towards

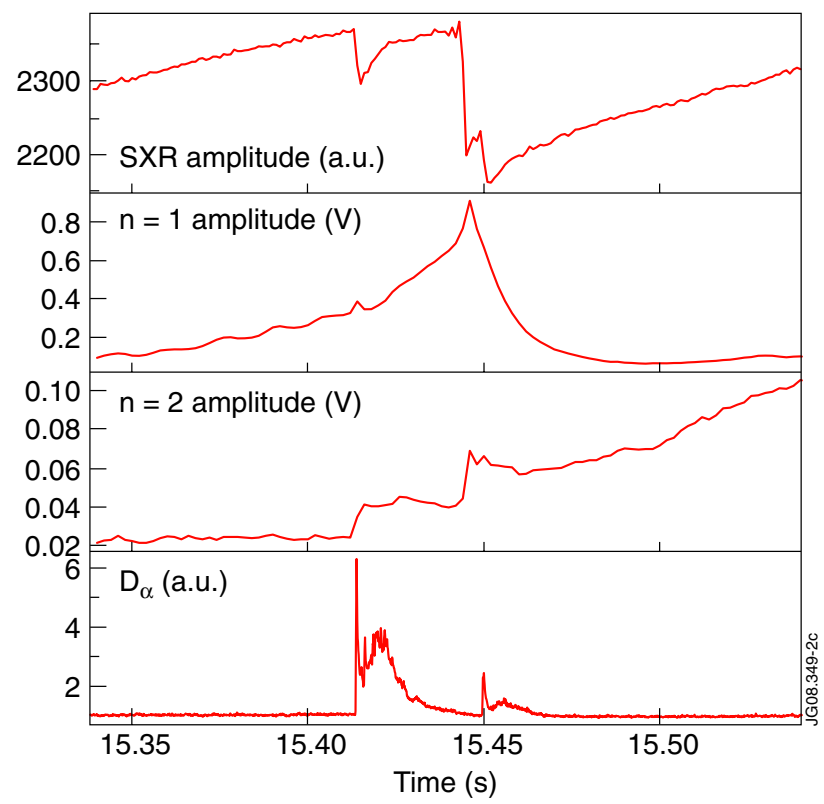

Figure 14. NTM onset due to ELM; soft-x-ray amplitude of central line-integrated signal, $n=1 \mathrm{MHD}$ activity, $n=2 \mathrm{MHD}$ activity, $\mathrm{H}_{\alpha}$ light in divertor: ELM at $15.413 \mathrm{~s}$; sawtooth crash at $15.45 \mathrm{~s}$ triggers $n=2$ activity $(3 / 2 \mathrm{NTM})$.

lower collisionality might be possible due to an increased pedestal temperature or density peaking. With an $H_{98(y, 2)}=$ 0.85 and $N^{\mathrm{GW}}=1$ a fusion power amplification of close to 10 should be possible with $15 \mathrm{MA}$ operation. For $17 \mathrm{MA}\left(q_{95}=\right.$ 2.6) the extrapolations with the code show the compatibility of those strongly radiating type-III ELMy H-modes with a power amplification in excess of 10 (see figure 17). In both cases, $15 \mathrm{MA}$ and $17 \mathrm{MA}$, the plasma core pollution is below $Z_{\text {eff }}=1.5$ (see figures 16 and 17). This does not include any contribution from the Be-wall. Assuming a core concentration of $2 \%$ of Be in the plasma core would then increase the $Z_{\text {eff }}$ to about 1.7, consistent with former ITER predictions [11].

\section{Summary and conclusion}

A large database on radiative type-III ELMy H-mode at JET does allow a reasonable extrapolation to ITER. The operational domain of the radiative type-III ELMy H-mode obtained by nitrogen seeding has been extended to low collisionality at JET. High density operation $\left(N^{\mathrm{GW}} \geqslant 0.9\right)$ at collisionalities only a factor of 3.5 higher than the ITER value with radiative power fractions of $70 \%$ and mild ELM activity has been demonstrated at JET.

Within the error bars of the data and based on the simplicity of the extrapolation models the extrapolation to ITER does allow the following statements: (a) the confinement (transport) should be sufficient to reach $Q=10$ at $17 \mathrm{MA}$ depending on point $\mathrm{d}$; (b) the steady-state heat load will be reduced to acceptable values; (c) the transient heat load should be acceptable even with respect to the most stringent limits; (d) the plasma pollution and hence the plasma core dilution could be slightly too high, leading to some reduction in plasma performance; (e) the accessibility of the type-III ELMy regime at higher pedestal temperatures seems to be possible; operation 


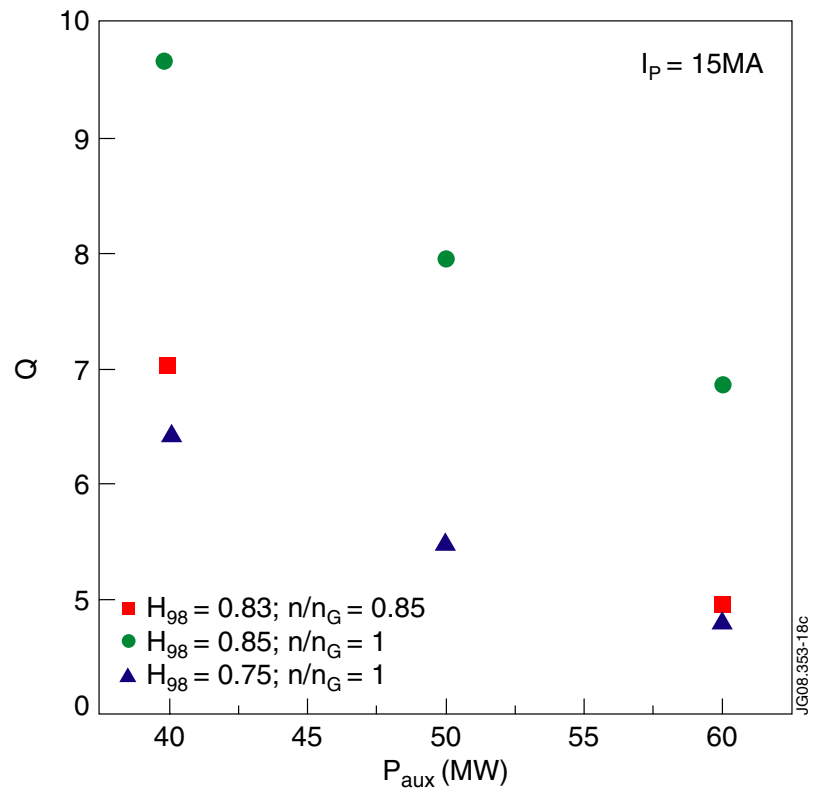

Figure 15. COREDIV: fusion amplification factor $Q$ versus the auxiliary heating power for the ITER 15 MA scenario for radiative scenarios with neon seeding.

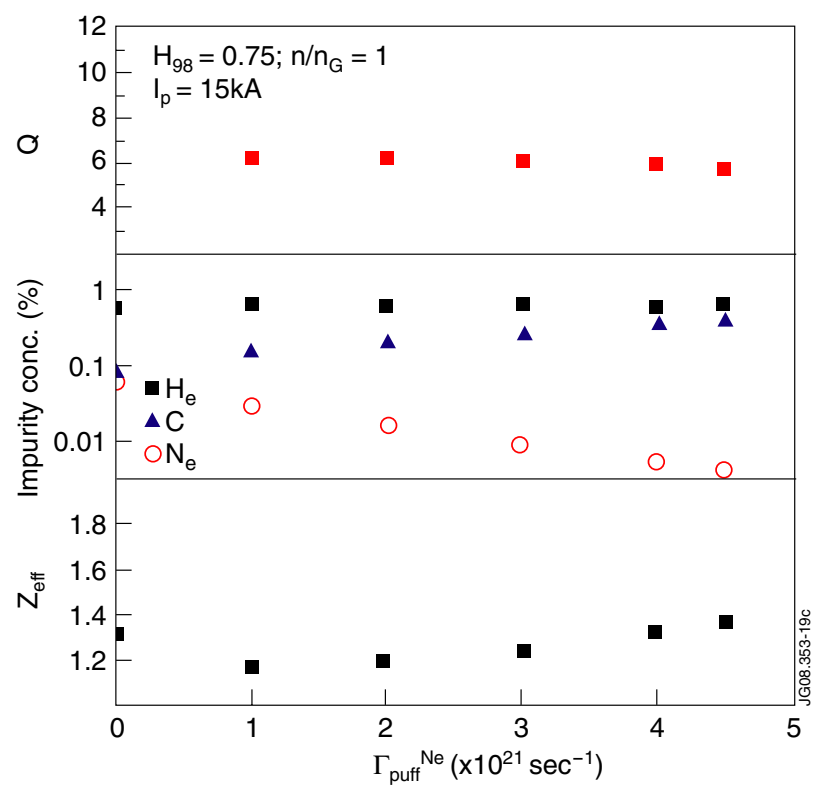

Figure 16. COREDIV: fusion amplification factor $Q$, impurity concentration in the core plasma and $Z_{\text {eff }}$ versus the neon gas fuelling rate for ITER $15 \mathrm{MA}$ scenario.

at low $q_{95}$ seems to be possible and reliable. It seems that the operation at low collisionality leads to slightly increased confinement, likely due to an increased pedestal temperature. Density peaking for type-III ELMy H-modes has been obtained at ITER relevant collisionality. However strong gas fuelling and operation at the Greenwald density might counteract the effect of density peaking.

Numerical simulations, benchmarked to the experimental results of the radiating type-III ELMy H-mode, predict with some uncertainties a possible operation scenario at $15 \mathrm{MA}$ with fusion amplification factors close to 10 .

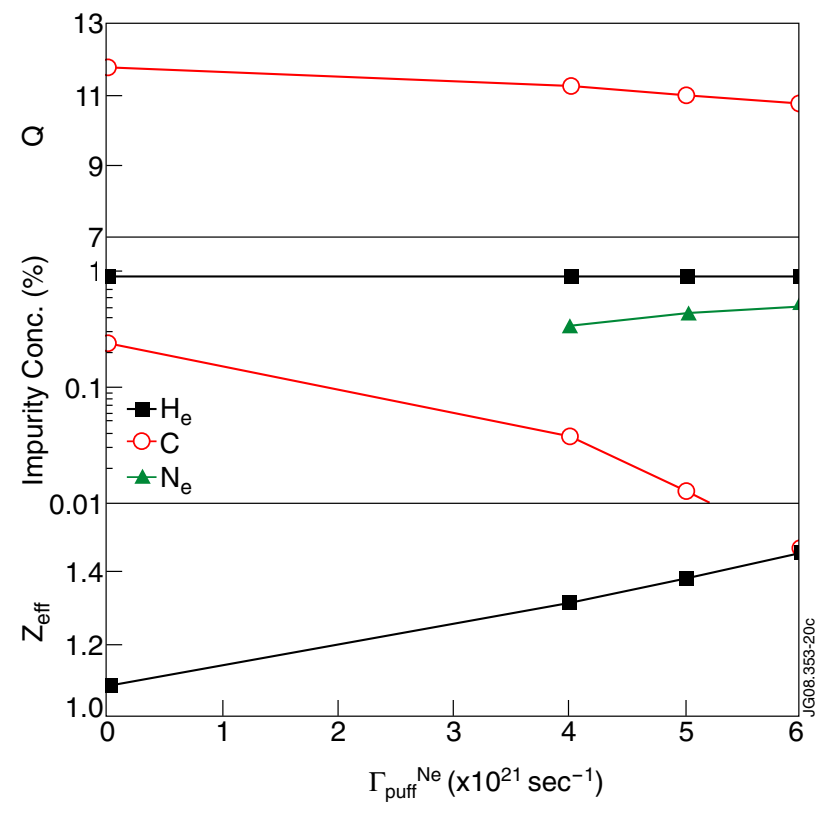

Figure 17. COREDIV: fusion amplification factor $Q$, impurity concentration in the core plasma and $Z_{\text {eff }}$ versus the neon gas fuelling rate for ITER $17 \mathrm{MA}$ scenario. Figure courtesy from [41].

If the confinement time of future ITER hybrid discharges (presently foreseen at low plasma current $I_{\mathrm{p}} 14 \mathrm{MA}$ ) is high enough to allow type-III ELMy operation with acceptable fusion performance $(Q \geqslant 5)$, then the experimental procedure described here can be envisaged to control the edge plasma conditions and get sustainable heat load (compatible with the ITER walls) without modifying the core $q$-profile, and thus the high $\beta_{\mathrm{N}}$ capability of the hybrid scenario (optimized for current drive sources and non-inductive current bootstrap). The relatively high impurity content and the extrapolation to ITER remain important issues to demonstrate the viability of the hybrid type-III ELM scenario as an integrated scenario for ITER. The use of the real-time control, maximization of confinement as observed in ASDEX Upgrade [44] and impurity decontamination techniques will be essential to improve the performances and the reliability of the scenario.

\section{Acknowledgments}

This work, supported by the European Communities under the contract of Association between EURATOM/FZJ, was carried out within the framework of the European Fusion Development Agreement. The views and opinions expressed herein do not necessarily reflect those of the European Commission.

Euratom $@ 2009$.

\section{References}

[1] Thomas P.R. 2008 Proc. 22nd Int. Conf. on Fusion Energy 2008 (Geneva, 2008) (Vienna: IAEA) IT/1-5 http://wwwpub.iaea.org/MTCD/Meetings/FEC2008/it_1-5.pdf

[2] Zohm H. et al 1996 Plasma Phys. Control. Fusion 38105

[3] Wagner F. et al 1982 Phys. Rev. Lett. 191408

[4] Federici G. et al 2003 Plasma Phys. Control. Fusion 451523

[5] Loarte A. et al 2003 J. Nucl. Mater. 313-316 919

[6] Osborne T.H. et al 1998 Plasma Phys. Control. Fusion 40845 
[7] Rapp J. et al 2002 Plasma Phys. Control. Fusion 44639

[8] Rapp J. et al 2004 Nucl. Fusion 44312

[9] Ingesson L.C. et al 2003 J. Nucl. Mater. 313-316 1173

[10] Jachmich S. et al 2002 Plasma Phys. Control. Fusion 441879

[11] Shimada M. et al 2000 J. Plasma Fusion Res. Ser. 377

[12] Greenwald M. et al 1988 Nucl. Fusion 282199

[13] Doyle E.J. et al 2007 Nucl. Fusion 47 S18

[14] Wesson J. 1987 Tokamaks (Oxford: Clarendon Press)

[15] Rapp J. et al 2005 J. Nucl. Mater. 337-339 826

[16] Joffrin E. et al 2005 Nucl. Fusion 45626

[17] Monier-Garbet P. et al 2005 Nucl. Fusion 451404

[18] Eich T. et al 2009 J. Nucl. Mater. 390-391 760

[19] Cordey J.G. et al 2002 Plasma Phys. Control. Fusion 441929

[20] Matthews G.F. et al 1999 Nucl. Fusion 3919

[21] Lomas P.J. et al 2000 Plasma Phys. Control. Fusion 42 B115

[22] Saibene G. et al 2002 Plasma Phys. Control. Fusion 441769

[23] Dumortier P. et al 2002 Plasma Phys. Control. Fusion 441845

[24] Kallenbach A. et al 1995 Nucl. Fusion 351231

[25] Cordey J.G. et al 2005 Nucl. Fusion 451078

[26] Igitkhanov Y. et al 1998 Plasma Phys. Control. Fusion 40837

[27] Igitkhanov Y. et al 2000 Contrib. Plasma Phys. 40368
[28] Sartori R. et al 2004 Plasma Phys. Control. Fusion 46723

[29] Chankin A.V. and Saibene G. 1999 Plasma Phys. Control. Fusion 41913

[30] Valovic M. et al 2004 Plasma Phys. Control. Fusion 461877

[31] Weisen H. et al 2006 Plasma Phys. Control. Fusion 48 A457

[32] Rapp J. et al 1997 Plasma Phys. Control. Fusion 391615

[33] Rapp J. et al 2009 J. Nucl. Mater. 390-391 238

[34] Rapp J. et al 2008 Plasma Phys. Control. Fusion 50095015

[35] Unterberg B. et al 1997 Plasma Phys. Control. Fusion 39 B189

[36] Telesca G. et al 1997 J. Nucl. Mater. 241-243 853

[37] Sauter O. et al 2002 Phys. Rev. Lett. 88105001

[38] Koslowski H.R. et al 2000 Nucl. Fusion 40821

[39] Nave M.F.F. et al 2003 Nucl. Fusion 431204

[40] Corre Y. et al 2008 Plasma Phys. Control. Fusion 50

[41] Zagorski R., Telesca G. and Rapp J. 2008 Contrib. Plasma Phys. 48179

[42] Zagorski R. and Stankiewicz R. 2003 J. Nucl. Mater. 313-316 899

[43] Roth J. et al 2004 Nucl. Fusion 44 L21

[44] Sips A.C.C. et al 2002 Plasma Phys. Control. Fusion 44 A 151 